Revista lus et Praxis, Año 26, № 2, 2020, pp. 55 - 77

ISSN $0717-2877$

Universidad de Talca - Facultad de Ciencias Jurídicas y Sociales

No es solo un robot: consideraciones en torno a una nueva personalidad jurídica y el redimensionamiento de las relaciones interpersonales Ana Karin Chávez Valdivia

Trabajo recibido el 6 de junio de 2019 y aprobado el 11 de septiembre de 2019

\title{
No es solo un robot: consideraciones en torno a una nueva personalidad jurídica y el redimensionamiento de las relaciones interpersonales
}

\author{
IT IS NOT JUST A ROBOT: CONSIDERATIONS REGARDING A NEW LEGAL PERSONALITY AND REMODELING INTERPERSONAL \\ RELATIONSHIPS
}

ANA KARIN CHÁVEZ VALDIVIA*

\begin{abstract}
RESUMEN
Presenciamos una nueva generación de sistemas robóticos que nos sitúa en un escenario en el cual las relaciones sociales se desarrollan en interacción con sistemas tecnológicos inteligentes diseñados para interactuar de forma personalizada cuando nos encontramos en situaciones que evidencian nuestra naturaleza más humana, vulnerable y sensible.

Durante mucho tiempo la fabricación y el diseño de robots enfrentaron tan solo a la posibilidad de demandas por responsabilidad del producto. Ahora sin embargo; el desarrollo de los robots sociales plantean la necesidad de una nueva categoría de sujeto jurídico con todo lo que ello significaría. Este nuevo escenario aunado al hecho de que el próximo paso en la tendencia será sin lugar a dudas el diseño y la construcción de robots "partners" suficientemente humanos y atractivos en múltiples aspectos que no solo dificultarán el poder distinguirlos de un ser humano a simple vista sino que también elevará nuestro nivel de percepción de tan solo criaturas no biológicas a criaturas que son mentales, sociales y morales; conllevará a replantearnos las formas más tradicionales del Derecho y nos enfrentará a uno de los cambios sociales más cuestionables de todos los tiempos.
\end{abstract}

ABSTRACT

We are attending a new generation of robotic systems which places us in a context where social relationships are developed in interaction with intelligent technological systems designed to interact in a very personalized way specially when we find ourselves in vulnerable or sensitive situations according to our human nature. For a long time, the manufacture and design of robots dealt with the possibility of claims for product liability. However; currently the development of social robots considers the need of a new category of legal subject with all that it involves. This new context combined with the fact that the next step in the tendency will be undoubtedly the design and construction of "partners" robots sufficiently human and attractive in numerous aspects that will not only make difficult to distinguish them from a human being at first sight but they will also raise our level of perception from only non-biological creatures to creatures which are mental, social and moral; this reality will make us reconsider the most traditional figures of Law and it will confront us to one of the most questionable social changes of all times.

PALABRAS CLAVE

Inteligencia artificial, personalidad jurídica de los robots, agente moral, robots sociales, relaciones interpersonales.

\section{KEY WORDS}

Artificial intelligence, legal personality of robots, moral agent, social robots, interpersonal relationships.

\footnotetext{
*Abogada. Doctora en Derecho y Magister en Derecho de la Empresa por la Universidad Católica de Santa María. Especialista en Derecho Informático y Gobierno Electrónico por la Universidad Inca Garcilazo de la Vega. Conciliadora Extrajudicial. Maestrista en Ia Maestría de las Nuevas Tecnologías de la Información y las Comunicaciones. Instituto Europeo Campus Stealle. Directora del Departamento Académico de Ciencias Jurídicas. Docente Asociada e investigadora de la Facultad de Derecho de la Universidad La Salle de Arequipa, Perú. Correo electrónico: achavez@ulasalle.edu.pe.
} 


\section{Introducción}

Si nos preguntamos la razón por la cual el binomio Robótica e Inteligencia Artificial (IA) genera tanta polémica, probablemente las primeras respuestas se orientarán hacia cuestiones intelectuales compuestas de temores fundamentados en amenazas a la subsistencia humana en sus diversas formas; al tiempo que, el avance tecnológico desdibujará cada vez más los límites entre ambas probablemente como resultado de lo que Calo denominó "un continuo" entre "robots" e "inteligencia artificial"; ya que al igual que Internet, los robots y otras entidades interactivas no tienen que diseñarse de ninguna manera en particular y debido a que existe un continuo de diseños potenciales y una variedad de usos potenciales diferentes, también hay un continuo de efectos potenciales que pueden tener estas nuevas tecnologías.

Es innegable que las implicancias y efectos de esta conjunción revolucionarán las estructuras sociales existentes y comportarán innumerables desafíos regulatorios que los actuales marcos legales - en su mayoría - no están preparados para enfrentar.

Sin embargo y de manera contradictoria esta dupla tecnológica se ha convertido no solo en un factor esencial sino en gran medida indispensable para el despliegue de prácticamente todas nuestras actividades habituales. En este contexto no es de sorprender que en los últimos años se estén desarrollando relaciones sociales con los robots en sus diversas manifestaciones.

El presente estudio considera la revisión de un largo proceso de evolución doctrinal donde la mayoría de ordenamientos reconoce la existencia de dos personalidades jurídicas diferenciadas, la de las personas individuales y la de las personas colectivas; así como las principales tendencias del estadio tecnológico actual que plantean la imperiosa necesidad de abordar la procedencia o no de crear una personalidad jurídica específica para los robots así como un agente moral artificial con todas las implicancias que esto significaría. Al mismo tiempo se constituye en un intento por hacer un análisis del redimensionamiento de nuestras relaciones interpersonales basadas en la naturaleza humana y su tendencia a crear vínculos emocionales y la viabilidad de que las relaciones humanorobot alcance otro nivel.

\section{Robots: Consideraciones en torno a una personalidad jurídica propia}

Si bien en su momento, producto de largos debates doctrinarios la mayoría de Sistemas Normativos reconocieron la existencia dos personalidades jurídicas diferenciadas; la evolución tecnológica ha hecho que en los últimos años el tema de la personalidad del robot haya pasado a un primer plano en la robótica social gracias a diversas publicaciones que abordan este planteamiento desde múltiples aristas ${ }^{1}$.

Aceptar la posibilidad de que los robots estén dotados de algún tipo de personalidad artificial implica un proceso de transición que no será el igual en todos los países ni encontrará el mismo nivel de aceptación debido a innumerables factores - sin perjuicio del interés teórico y filosófico que implica abordar la creación de una nueva Personalidad Jurídica - pero que aun siendo así, todas las sociedades deberán afrontar en un corto periodo de tiempo. El debate ya se inició y una vez superada la infinidad de cuestionamientos y controversias que ha suscitado; el siguiente paso será determinar qué postura asumiremos; con todo lo que ello implique.

\footnotetext{
${ }^{1}$ Dentro de los principales exponentes podemos mencionar: Steffen Wettig y Eberhard Zehendner (2003); Samir Chopra y Laurence White
} (2011); Patrick Hubbard (2010); Rob Sparrow (2012). Kamil Muzyka (2014) y Evan Joseph Zimmerman (2015). 
La tesis de la voluntad propugnada por Savigny - como esencia del derecho subjetivo y de la personalidad jurídica; y como facultad de proponerse fines y realizarlos - estaría en la actualidad superada como elemento básico para definir y reconocer la realidad de la persona jurídica. No obstante, esta tesis parece recobrar cabida a la hora de configurar una personalidad jurídica para los robots, tal como se evidencia de la Resolución del Parlamento de la Unión Europea ${ }^{2}$ cuando conmina a la Comisión para que la personalidad jurídica se otorgue únicamente a aquellos entes capaces de tomar decisiones autónomas ${ }^{3}$, lo que podría significar una clara reminiscencia a las tesis de Savigny; entendiendo como robot dotado de voluntad aquel que pueda tomar decisiones autónomas inteligentes, esto es, actuar sin una indicación ajena previa, conforme a un discurrir lógico propio ${ }^{4}$.

Así mismo, cabe señalar que el Parlamento en sus recomendaciones evita usar la palabra "voluntad" haciendo constante alusión al término autonomía - el mismo que desde la época griega ya hacía referencia a cierto estado de "capacidad" o de "voluntad propia" y que posteriormente con Kant ${ }^{5}$ alcanza una definición más clara en la filosofía-. Sin embargo; y no obstante, cabe mencionar que en la edad media la voluntad recibió diversas nomenclaturas entre las que debe destacarse el apetito racional y el apetito intelectivo, elementos estos que vincularían por tanto la autonomía con la inteligencia (artificial).

Siendo así, en el campo de la Robótica la "autonomía" se referiría a la posibilidad de adoptar decisiones por uno mismo, sin dejarse influenciar por los demás; y en la previsión del Parlamento Europeo, es la capacidad de ser responsable del propio comportamiento y de conducir la existencia; de acuerdo con dictados propios ${ }^{6}$.

Señala Peter Singer ${ }^{7}$ que la autonomía es condición necesaria para afirmar que un individuo es un agente moral, teniendo en cuenta que por "autonomía" se entiende la capacidad de elegir, de hacer y actuar según las propias decisiones".

Es evidente que el solo hecho de considerar la posibilidad que un robot pueda alcanzar el estado de agente moral implica un cambio total de paradigmas normativos al tiempo que cambiaría la forma en que se usa y diseña esta tecnología.

Kaplan ${ }^{8}$ por su parte refiere que una descripción ampliamente aceptada de los agentes morales es que estos deben de ser capaces de dos cosas: una de ellas es poder percibir las consecuencias morales correspondientes de sus acciones y la otra elegir entre las vías de acción pertinentes. Precisa además el autor que ninguno de esos dos requisitos depende de un juicio subjetivo e innato de lo correcto y lo incorrecto ya que simplemente los agentes tienen que ser

\footnotetext{
2 PARLAMENTO EUROPEO (2017).

${ }^{3}$ El Parlamento Europeo en el punto 59 de su Resolución pidió a la Comisión que cuando realizara una evaluación de impacto de su futuro instrumento legislativo, explorara, analizara y considerara las implicaciones de todas las posibles soluciones jurídicas, tales como (...) f) crear a largo plazo una personalidad jurídica específica para los robots, de forma que como mínimo los robots autónomos más complejos puedan ser considerados personas electrónicas responsables de reparar los daños que puedan causar, y posiblemente aplicar la personalidad electrónica a aquellos supuestos en los que los robots tomen decisiones autónomas inteligentes o interactúen con terceros de forma independiente.

${ }^{4}$ La Real Academia Española emplea diversas acepciones para definir la palabra voluntad entre ellas, cabe destacar aquella que la conceptualiza como la facultad de decidir y ordenar la propia conducta, la elección de algo sin precepto o impulso externo que a ello obligue o la elección hecha por propio dictamen o gusto, sin atención a otro respeto o reparo. REAL ACADEMIA ESPAÑOLA (2019a).

${ }^{5}$ Immanuel Kant entiende por "autonomía de la voluntad" la "constitución de la voluntad, por la cual es ella para sí misma una ley independientemente de cómo estén constituidos los objetos del querer-. El principio de la autonomía es pues, no elegir de otro modo sino de éste". KANT (1922), p. 78.

${ }^{6}$ Concordamos con Ercilla cuando señala que la "autonomía" como tal se refiere a la predisposición de actuar en un entorno y no a una libertad absoluta de proceder conforme a dictados morales de elaboración propia; debido a que los mismos, serían dados por los humanos. ERCILLA (2018), p. 17.

${ }^{7}$ SINGER (2011), p. 84.

${ }^{8}$ KAPLAN (2017), p. 53.
} 
capaces de controlar sus propias acciones y valorar las consecuencias de las mismas frente a una normas morales objetivas; siendo irrelevante si la norma se genera a sí misma, si ellos comprenden la teoría que sirve de base al a norma, si la aceptan o no, o si pueden percibir la diferencia entre la rectitud moral y el pecado.

En consecuencia, un intelecto sintético - como lo denomina Kaplan- puede cumplir los requisitos de responsabilidad moral y consecuentemente clasificar como agente moral en la medida que posea la suficiente capacidad para sentir los aspectos moralmente relevantes de su entorno y pueda elegir sus acciones.

No obstante, para que esto sea posible debemos aceptar que la programación del robot será el factor determinante en toda clase de decisiones que este pueda llegar a tomar. No podremos esperar que un robot tome una decisión correcta sin ningún tipo de orientación previa y es probable que en este punto radique uno de los mayores problemas.

A lo largo del tiempo se han esgrimido innumerables teorías y argumentos que han dado como resultado las más diversas posturas en relación tan solo a la idea de lo que se entiende por "moral"; en consecuencia; si no existe un consenso aceptado por los expertos sobre como tendría que ser un código moral entre nosotros mismos como seres humanos, difícilmente los humanos podrán dotar a una inteligencia artificial de una programación de contenido moral de aceptación unánime; por lo que el tema indiscutiblemente trasciende a una simple cuestión de ingeniería.

El ser humano - al menos por ahora- deberá programar un código de ética, es decir, deberá crear sistemas robóticos éticos, pero la interrogante continúa en torno al cómo lograr en otros lo que en siglos como humanidad no hemos logrado entre nosotros mismos. Probablemente, en un futuro los robots entre ellos mismos lo consigan y trasciendan al imperativo categórico Kantiano, la banalidad del mal de Arendt, el utilitarismo de Bentham, el saber como fin último de Aristóteles o la ética de Nietzsche; y tal vez la propuesta de Yudkowski viabilice esta trascendencia, ya que la volición coherente extrapolada para lograr una inteligencia artificial amigable busca que los sistemas aprendan la parte de la voluntad colectiva que es coherente, no divergente dando como resultado que el principio ético a programar sea el que lleve a decisiones colectivas acordadas, es decir, no programar principios éticos definidos por humanos, sino dejar que los métodos matemáticos que ya conocemos se encarguen de sintetizar esos criterios.

Yudkowsky ${ }^{9}$ señala que no podemos estar seguros sobre la naturaleza de la ética, por esta razón una IA completamente amigable necesita ser capaz de manejar literalmente cualquier pregunta moral o ética que un humano pudiera responder y para lograr esto requiere tener el conocimiento de cada factor que contribuya a la ética humana.

Este contexto trae consigo otro cuestionamiento que gira en torno a determinar si existiría alguna razón válida por la que no se pudiera permitir que la inteligencia artificial redefina la ética.

Sin embargo, son por ahora los humanos, los agentes rectores responsables de la acción de sus inteligencias artificiales sensibles o racionales, de modo que cualquier abuso de la IA haría a los humanos moral y legalmente culpables.

En este sentido para garantizar un conjunto común de valores para la producción de robots en Setiembre del 2010 se reúnen el EPSRC (Engineering and Physical Sciences Research Council) y el AHRC (Arts and Humanities Research Council) congregando diversos expertos en las áreas de tecnología, industria, arte, derecho y ciencias sociales para discutir sobre robótica, sus aplicaciones en el mundo real y la infinidad de promesas que ofrece para el beneficio de la sociedad ${ }^{10}$.

\footnotetext{
${ }^{9}$ YUDKOWSKY (2001), p. 15.

${ }^{10}$ ENGINEERING AND PHYSICAL SCIENCES RESEARCH COUNCIL (2010).
} 
Producto de esta reunión se presentó una versión semi-legal de un conjunto de reglas denominadas "Principios para los diseñadores, creadores y usuarios de robots" si bien la comisión señaló que se constituye en una normativa un poco imprecisa, es fácil de expresar y logra recoger el sentido de fondo para una audiencia no especializada debido a los comentarios presentados en torno a cada principio. Así tenemos:

1.- Los robots son herramientas de múltiples usos. Lo robots no deben ser diseñados exclusiva o principalmente para matar o dañar a los seres humanos, salvo que exista un interés nacional.

2.- Los seres humanos y no los robots son los sujetos responsables. Los robots son herramientas diseñadas para cumplir con el Derecho y los derechos fundamentales, entre los que está la privacidad. 3.- Los robots son productos. Deben ser diseñados utilizando procesos que aseguren su protección y seguridad.

4.- Los robots son artefactos fabricados. No deben ser diseñados de manera engañosa para explotar a usuarios vulnerables, sino que su naturaleza como máquina debe ser transparente.

5.- Siempre debe ser posible saber quién es legalmente responsable por el comportamiento de un robot.

Adicionalmente a estos principios se esbozaron algunos mensajes diseñados para motivar la responsabilidad dentro de la investigación de la robótica y la comunidad industrial y generar confianza en el trabajo que realizan.

Por otro lado el documento denominado "European Civil Laws in robotics" del mes de octubre del 2016 elaborado por la Eurocámara ${ }^{11}$ propone nueve leyes en torno al tema en cuestión, que en síntesis establecen:

1. Proteger a los seres humanos de los daños causados por robots.

2. Respetar el rechazo de la atención por parte de un robot. Este principio sigue al primero y establece el derecho de una persona a negarse a ser cuidado por un robot.

3. Proteger la libertad humana frente a los robots.

4. Proteger a la humanidad contra las violaciones de la privacidad cometidas por un robot.

5. Gestión de datos personales procesados por robots.

6. Proteger a la humanidad contra el riesgo de manipulación por robots.

7. Evitar la disolución de los vínculos sociales.

8. Igualdad de acceso al progreso en robótica.

9. Restringir el acceso humano a las tecnologías de mejora.

Si comparamos las leyes de Asimov con los principios de la robótica establecidos por el EPSRC y el AHRC y las nueve leyes europeas podemos observar notables diferencias. Al mismo tiempo, podrían dar lugar a múltiples cuestionamientos por lo que su aplicación en un momento determinado probablemente no sería tan sencilla como su enunciado, lo que nos conduciría a

\footnotetext{
${ }^{11}$ Con vistas a la evolución de la robótica y la inteligencia artificial, la Comisión de Asuntos Jurídicos consideró oportuno que la Unión Europea adoptara medidas respecto de las cuestiones jurídicas y éticas planteadas por estas nuevas tecnologías. Con este fin, la Comisión JURI creó un grupo de trabajo en 2015 con el objetivo principal de elaborar normas de derecho civil "europeas" en este ámbito (lege ferenda). A pesar de respetar el derecho de iniciativa de la Comisión Europea, el 31 de mayo de 2016 este grupo presentó un proyecto de informe en el que se establecen una serie de recomendaciones sobre las normas de derecho civil en materia de robótica. Este proyecto incluye una propuesta de resolución del Parlamento Europeo, acompañada de un anexo con recomendaciones detalladas sobre el contenido de una posible propuesta legislativa. También incluye una exposición de motivos que señala que el objetivo del futuro instrumento es establecer los "principios generales y éticos que rigen el desarrollo de la robótica y la inteligencia artificial con fines civiles".
} 
reflexionar hasta qué punto y en qué medida los robots podrían llegar a convertirse en "agentes morales" al tener que enfrentar los mismos dilemas morales de un ser humano.

Como acertadamente señala Hutan Ashrafian ${ }^{12}$ si bien todas estas regulaciones propuestas llevan consigo los ideales de la futura actividad potencial de los robots entre la humanidad, no consideran la naturaleza de cómo actuará cada inteligencia artificial sobre otras inteligencias artificiales. El autor se cuestiona sobre cuál es el carácter de la interacción robot-robot y qué moral debería demostrar esta interacción y señala que existe una necesidad de investigación ética en esta área, especialmente en la medida en que AlonAI (Inteligencia Artificial sobre Inteligencia Artificial) pueda impactar no solo la interacción humano-robot (HRI) sino también las interacciones entre los seres humanos.

Por lo pronto, y acorde al estado de las cosas cabe señalar que si bien los sistemas jurídicos actuales aún no consideran a un robot responsable en sí mismo de los actos u omisiones que causen daño a terceros y las normas en materia de responsabilidad civil se aplican al fabricante, propietario o usuario según fuere el caso -es decir el sistema considera hasta ahora al robot como una simple herramienta que se convierte a lo mucho en un instrumento que reflejaría los intereses morales de otros - la innegable evolución tecnológica nos conduce a considerar la posibilidad real aunque potencialmente distante aún - sobre todo en los país en vías de desarrollo- de que los robots lleguen a convertirse en "agentes morales artificiales".

Por consiguiente, y retomando a Savigny cuando considera que las personas morales son el resultado de un artificio legal que la ley elabora por razones de conveniencia - recurriendo entonces a la ficción - compartimos la opinión de Ercilla García ${ }^{13}$ al precisar que devendría en una cuestión de conveniencia la creación de una personalidad jurídica específica para los robots, en consideración a que un lapso de tiempo medianamente corto la sociedad contará con entes no humanos dotados de voluntad que realizarán actos susceptibles de crear derechos u obligaciones en el ámbito jurídico.

En este orden de ideas señala el autor que el panorama del sistema jurídico se conformaría por el reconocimiento de tres personalidades jurídicas; la del ser humano individual dotado de voluntad propia; la de las colectividades humanas dotadas de voluntad común producto de voluntades aisladas y la de los sistemas ciber-físicos dotada de una voluntad surgida de procesos algorítmicos.

Sostiene Ercilla también qué en virtud de la Teoría pura del Derecho, la persona jurídica, tanto individual, colectiva; como electrónica ${ }^{14}$, no serían sino un conjunto de obligaciones jurídicas y derechos subjetivos, cuya unidad se expresa metafóricamente en el concepto de "persona". Al tiempo que el concepto de "portador" de derechos y obligaciones jurídicas desempeña un papel decisivo en la teoría tradicional de la personalidad jurídica.

Si bien, determinar el contenido concreto de los derechos y obligaciones que esta "persona" haya de "portar" deviene en un tema crucial, complejo y altamente debatible, al no ser objeto de la presente investigación no será abordado a profundidad ${ }^{15}$; sin embargo, señalaremos algunas ideas que merecen ser objeto de reflexión. Si bien, Ercilla García considera algunos aspectos que serían necesarios contemplar en el intento por esbozar una personalidad jurídica específica para los

\footnotetext{
${ }^{12}$ ASHRAFIAN (2015), pp. 20-40.

${ }^{13}$ ERCILLA (2018), p. 17.

${ }^{14}$ Término empleado en PARLAMENTO EUROPEO (2017).

${ }^{15}$ No obstante, será necesario contemplar también una suerte de principios que sustenten la normativa en torno al tema y que podrían ser recogidos de las Leyes de Asimov, las de Clarke, los principios señalados el por Parlamento Europeo, u otros, en tanto sean pertinentes a la creación de un Corpus luris Robótico coherente y sistemático y en la medida que su enunciación haga posible una adecuada interpretación de carácter general.
} 
robots, en su mayoría estos son los señalados en la Recomendación del Parlamento Europeo; tenemos así: la Integridad, la capacidad, la culpa, la responsabilidad y el patrimonio, la tributación, la materialización del "Ius Patrimonii", la computación distribuida, la propiedad intelectual, el matrimonio y las obligaciones.

De los aspectos señalados abordaremos el sentido que el autor le concede a la integridad ya que lo consideramos un factor importante para nuestra propuesta. Refiere Ercilla que de existir la obligación legal de no dañar a las personas ciber-físicas les correspondería a estas un derecho subjetivo a su "integridad material". Es decir; La omisión a que un individuo estaría obligado frente a otro podría consistir en la omisión de determinada acción del propio obligado, como sería la obligación de no lesionar a otro individuo. Si se designa la relación de un individuo, que se encuentra obligado con respecto a otro a omitir determinada conducta como "derecho" entonces no es sino un reflejo de esa obligación. Este punto podríamos relacionarlo con la postura de Darling cuando hace referencia al "maltrato" y el planteamiento de Hutan Ashrafian ${ }^{16}$ por salvaguardar y ayudar a prevenir una potencial explotación y abuso a los cuales estará expuesta la IA producto de su interacción con el hombre.

En este orden de ideas nos atrevemos a proponer como primer derecho de los robots sociales: el derecho a la integridad y a no sufrir daño, derecho cuya naturaleza sería inalienable.

Cabe señalar que el daño en mención no necesariamente provendría exclusivamente de los seres humanos, sino también de otra IA - tal como acertadamente lo precisa Ashrafian-. Acorde al estado actual de las cosas - en algunos países más que en otros- deviene en un tema crucial salvaguardar a los robots sociales de la crueldad expresada a través de comportamientos vejatorios o humillantes de diversa naturaleza. El derecho que proponemos, por otro lado, guardaría congruencia con nuestra tendencia a proteger aquello con lo que nos relacionamos de manera permanente y cercana o con aquello hacia lo cual desarrollamos ciertos lazos que denotan diversos grados de intensidad. Consideramos además que en cierto punto no podremos negar que este derecho probablemente oculte la preocupación por proteger más nuestro propio estado emocional que cualquier otro criterio biológico objetivo; en consecuencia, reviste un carácter discutible al igual que todas las temáticas que pretendan abordar el binomio robótica inteligencia artificial.

Este planteamiento, sin embargo, nos lleva también a considerar la divergencia de contextos tecnológicos. Para la conciencia colectiva occidental la idea del "robot inteligente" provoca reacciones adversas, es más podría afirmarse que el vislumbrar una sociedad en la cual humanos y robots coexistan provoca cierto temor y hasta rechazo. La postura oriental es completamente contraria, su sociedad avanzó con una imagen muy positiva de los robots. Mas aún, según la visión shintoista japonesa de los robots, ellos, como todo lo demás, tienen un alma. A diferencia de Occidente, los robots no son vistos como criaturas peligrosas y naturalmente, pertenecen a los seres humanos. La literatura actual centra su atención en buscar la forma en como las distintas sociedades se protegerán de los robots que ellos mismos están creando. Los estados empiezan a preocuparse por regular a la inteligencia artificial en base a infinidad de temores aún infundados pero considerados previsibles.

No existe sin embargo, el mismo grado de preocupación en torno a los sistemas de protección que serán necesarios para salvaguardar a los robots de sus propios creadores. Y si bien algunos autores abordan el tema de los derechos de los robots no lo hacen en su mayoría desde una óptica exclusivamente centrada en el robot en sí mismo. En este contexto, Pagallo ${ }^{17}$ a la hora

\footnotetext{
${ }^{16}$ ASHRAFIAN (2015), pp. 20-40.

${ }^{17}$ PAGALLO (2013), p. 21.
} 
de abordar la sustantividad de un Derecho de los Robots pone de relieve como las tendencias en los derechos comparados pueden sistematizarse en tres grandes respuestas:

a) Una primera aproximación, que únicamente examina como los robots afectan a los conceptos y principios legales tradicionales, como por ejemplo es el caso del concepto de persona jurídica; personería abordada en la presente investigación.

b) Otra segunda dirección que pretende edificar una nueva disciplina jurídica con nuevos paradigmas, como sería el caso por ejemplo de la responsabilidad por operaciones realizadas por sistemas robóticos autónomos

c) Una tercera y última dirección, que por el contrario, niega su especificidad y convierte los retos que plantean los robots en cuestiones que se pueden abordar siempre dentro de los parámetros de los vigentes ordenamientos.

Por su parte Ryan Calo ${ }^{18}$ considera que un sistema actúa sobre su entorno en la medida en que cambia ese entorno de manera directa. Una tecnología no actúa y por lo tanto no es un robot, cuando simplemente proporciona información en un formato inteligible ya que debe ser de alguna manera.

Un robot en el sentido más fuerte y completo del término existe en el mundo como un objeto corporal con la capacidad física de un despliegue propio. Siendo así, es de particular interés para el análisis legal una definición funcional de lo que significa que la tecnología actúe, en lugar de que solamente informe.

El autor señala que para hablar de una tecnología transformadora - por la combinación que implica- debe tenerse presente las cualidades esenciales que la caracterizan como tal y que permiten al mismo tiempo ir más allá de una sola definición técnica de lo que sería un robot: objetos o sistemas artificiales que perciben, procesan y actúan sobre el mundo al menos en cierto grado.

Sostiene además que estas cualidades ya sea que se presenten de manera independiente o especialmente en combinación resultan relevantes para una variedad extraordinariamente amplia de contextos legales. Por esta razón consideramos de trascendental importancia referirlas en el mismo idioma en que fueron propuestas por el autor: embodiment, emergence y social valence. De otro lado retomarlas en el idioma original reviste una importancia trascendental para poder entender el sentido del estudio de Calo, ya que las interpretaciones o traducciones podrían resultar ser no solo un tanto engañosas sino también inducirnos a error en torno al real sentido de estas cualidades esenciales; más aún si las consideramos relevantes en el intento de establecer una nueva personalidad jurídica y consecuentes derechos atribuibles a esta.

Si bien Barrio ${ }^{19}$ denomina estas cualidades esenciales propuestas por Calo como propiedades privativas y las refiere como: corporeidad, impredecibilidad e impacto social; por las consideraciones previamente señaladas no compartimos los términos que emplea y el sentido que les atribuye.

Encontramos así, que la palabra embodiment ${ }^{20}$ difiere ampliamente de la palabra corporeidad $^{21}$. Al explicar Calo esta cualidad señala que los robots, difieren de las computadoras y el software precisamente en que están organizados para actuar sobre el mundo. Esta capacidad de

\footnotetext{
${ }^{18}$ CALO (2015), p. 531.

${ }^{19}$ BARRIO (2018), p. 96.

${ }^{20}$ El Diccionario de Cambridge define la palabra embodiment como: Someone or something that represents a quality or an idea exactly. CAMBRIDGE DICTIONARY (2019a).

${ }^{21}$ Este término no aparece como tal en el diccionario Cambridge, CAMBRIDGE (2019). En Collins sin embargo; lo encontramos como corporeal nature COLLINS DICTIONARY (2019a). De otro lado en el diccionario Linguee la palabra corporeidad no es traducción de "embodiment", sino de "corporeality". LINGUEE (2019).
} 
actuar físicamente sobre el mundo se traduce, a su vez; en el potencial de dañar físicamente a las personas o la propiedad. Consideramos que es precisamente esta capacidad con su consecuente potencial, los que se constituyen en la esencia del término en cuestión; es decir, embodiment no hace referencia al solo hecho de tener un cuerpo material como si lo hace el término "corporeidad", sino que trasciende al concepto limitativo de materialidad corpórea.

La segunda cualidad emergence ${ }^{22}$ no es igual que impredecibilidad ${ }^{23}$. El comportamiento emergente es un objetivo claramente establecido de la robótica y la inteligencia artificial, yendo directamente al componente "pensar". Además, debido a que un sistema emergente aprende del comportamiento anterior, mejorará en una tarea con el tiempo, incluso sin ayuda.

Agrega Calo que es importante destacar que el comportamiento emergente puede conducir a soluciones que ningún ser humano hubiera encontrado por sí solo. El autor cita a Stephen Johnson ${ }^{24}$ cuyos trabajos en relación a esta cualidad abarcan varias disciplinas. Johnson ve la esencia de la emergencia como el acoplamiento de la complejidad y la utilidad, el movimiento de reglas de bajo nivel a tareas de aparentemente alta sofisticación. Alan Turing entendió la utilidad de la emergencia y, según Johnson, los diseñadores contemporáneos de sistemas inteligentes confían en los principios de la emergencia con una frecuencia cada vez mayor.

Para Barrios la impredecibilidad - término que equipara a emergence-implica a su vez que el robot decide con cierta autonomía. Si aún en este punto concordáramos con Barrios, tendríamos que hacer la aclaración de que no se trata tan solo de autonomía en términos generales, sino de una autonomía por diseño.

La tercera cualidad a la que Calo denomina social valence ${ }^{25}$ tiene un contenido diferente al de impacto social.

Los robots también evocan respuestas en las personas. Calo señala que el psicólogo Peter Kahn y sus colegas llevaron a cabo una serie de experimentos para intentar comprender cómo pensamos acerca de los robots. Los resultados llevaron al equipo a formular la hipótesis que los robots pueden pertenecer a una "categoría ontológica" completamente nueva. Los sujetos no tienden a pensar en robots personificados como vivos, pero tampoco los consideran objetos. Más aún, en los sujetos de tales estudios hubo tendencia a atribuir estados mentales a los robots y les fue difícil participar de determinados comportamientos, como por ejemplo, provocar incomodidad, lo que hubiera sido fácil de haberse tratado de un objeto.

Por otro lado refieren Groom et al. ${ }^{26}$ que el grado de antropomorfismo en forma robótica afecta la manera en que las personas interactúan con los robots. Precisa que la investigación ha demostrado: Que se elogia más a los robots antropomórficos y se castiga menos en las interacciones colaborativas entre el equipo humano y el robot. Que las formas más antropomórficas de robots también aumentan los sentimientos de utilidad y comprensión; y que se percibe que las formas antropomórficas de robots son más comprensivas, amigables e inteligentes que los robots funcionales.

\footnotetext{
${ }^{22}$ El Diccionario de Cambridge define la palabra emergence como: the fact of something becoming known or starting to exist. CAMBRIDGE DICTIONARY (2019b).

${ }^{23}$ Esta palabra como tal no la encontramos en el Diccionario de Cambridge; lo mas cercano al término es la palabra imperdecible; conceptualizada de la siguiente forma: unpredictable, changing often so that it is posible to know what will happen next. CAMBRIDGE DICTIONARY (2019c). Por su parte el diccionario. De otro lado, Collins conceptúa la palabra impredecibilidad como unpredictability. COLLINS DICTIONARY (2019b).

${ }^{24}$ CALO (2015), p. 539.

${ }^{25}$ Business dictionary define el término como: Negative or positive psychological value assigned by a person to another person, event, goal, job, object, outcome, etc., based on its attractiveness to him or her. BUSINESS DICTIONARY (2019).

${ }^{26}$ GROOM et al. (2009), p. 32.
} 
Aunado a todas las consideraciones señaladas no debe olvidarse que el Derecho es respuesta a realidades sociales y estas no permanecen inmutables en el tiempo; en consecuencia, el Derecho no puede permanecer ajeno a cambios sustanciales que demandan su obligada atención. En este contexto, Dario et al. ${ }^{27}$ en "Robot Companions for Citizens" - propuesta que data del 2011abordó temáticas que años más tarde recogería la resolución del Parlamento Europeo. EI documento en mención parte del hecho que el envejecimiento de la población generará nuevas necesidades asistenciales y de salud en una sociedad, como la europea, que, según sus estimaciones en un horizonte de 40 años, tendría una población cuyo 35\% superaran los 60 años. Esta realidad es también compartida por la sociedad japonesa; en la cual, no solo se espera que los robots reemplacen la necesidad de inmigrantes y trabajadores migrantes, sino que los humanoides están siendo diseñados para cumplir muchos roles, incluyendo la preservación de costumbres japonesas "únicas" y formas de arte escénico tradicionales.

En este sentido, señala Jennifer Robertson ${ }^{28}$ que existe un creciente apoyo popular, por un lado, para denegar los derechos civiles a los residentes permanentes por el otro, para conferir los derechos de ciudadanía y residencia a los robots y animales no humanos, e incluso Personajes de caricatura.

Refiere la autora que la población y la fuerza de trabajo de Japón está envejeciendo y disminuyendo rápidamente. Así, en el 2014, la tasa de natalidad era de 1.3 hijos por mujer casada, y alrededor del 25 por ciento de la población de aproximadamente 127.3 millones de personas (que incluía alrededor de 2 millones de residentes legales extranjeros) era mayor de 65 años; se espera que ese porcentaje aumente en 2050 a más del 40 por ciento. Las estimaciones del Ministerio de Salud, Trabajo y Bienestar indican que la población disminuirá a menos de 111 millones en 2035 y a menos de 90 millones en 2055. En pocas palabras, las mujeres y los hombres posponen el matrimonio hasta casi los 20 años y los primeros 30 años, y algunos están evitando por completo el matrimonio. Incluso las parejas casadas optan por no tener hijos; hoy en día, las mascotas de las casas superan en número a los niños.

De otro lado, señala Robertson que las encuestas nacionales realizadas por la Oficina del Gabinete indican que los ciudadanos japoneses no se sienten cómodos con la idea de ser atendidos por enfermeras y cuidadores extranjeros, así como que muchas personas mayores se preocupan particularmente por el estrés de lidiar con las diferencias lingüísticas y culturales; de ahí que se sienten más cómodos compartiendo entornos de vida con robots al punto que más del $80 \%$ manifiesta interés por adquirir un robot cuidador. Esta misma tendencia se refleja en el ámbito laboral donde prefieren tener robots que contratar trabajadores migrantes.

Desde el año 2007, el estado japonés ha promovido activa e incansablemente una sociedad y un estilo de vida dependientes del robot; tal es así que, en febrero de ese mismo año, el primer ministro Shinzð Abe dio a conocer "Innovation 25", un plan visionario para revitalizar la economía, la sociedad civil y los hogares "tradicionales" de Japón para el año 2025.

Ante la evolución y tendencias de los diversos contextos sociales, la propuesta "Robot Companions for Citizens" precisa la necesidad de atender situaciones actuales por lo que los Compañeros Robots serán una nueva generación de máquinas que ayudarán y asistirán principalmente a las personas mayores en las actividades diarias del hogar, en su lugar de trabajo y en otros entornos; señala además el documento, que estos compañeros serán máquinas fuertes,

\footnotetext{
${ }^{27}$ DARIO et al. (2011), pp. 47-51.

${ }^{28}$ ROBERTSON (2014), p. 578.
} 
agraciadas y suaves; y sensibles — sensibilidad que deviene en la integración de la percepción, conocimiento, comunicación, sentimiento, acción conciencia de sí mismo de otros y del entorno-.

En este orden de ideas es indiscutible que la sociedad deberá lidiar con robots dotados de voluntad, aprendizaje e inteligencia artificial (IA) que dará como resultado que cada ente electrónico posea una individualización diferenciada de los demás una vez fabricado y programado; por lo que estarán en capacidad de distinguirse de los demás en la medida que sean capaces de aprender de su entorno y en consideración a las circunstancias alrededor de las cuales transcurra su existencia. Todo esto conllevará a que por ser seres autónomos relacionables con la sociedad precisen de un estatus jurídico distinto ${ }^{29}$.

Sin embargo; cabe mencionar que evidentemente no todas las IA se encuentran en el mismo nivel desarrollo. Si bien es cierto John Searle ${ }^{30}$ construyó la distinción entre la IA débil y la IA fuerte hacia 1980 su posición fue enfrentada por quienes sostienen la inespecificidad humana de la inteligencia y cuestionaron el enigma de la habitación china.

Una de las áreas del Instituto Max Planck de Investigación en Ciencias de la Computación denominada Robótica y Sistemas cyber-físicos textualmente prescribe ${ }^{31}$ :

Robots and other complex cyber-physical systems (CPS) sense, process, and react to information from the physical world. They must operate safely even in the presence of uncertainties and resource constraints. To enable advanced robotics and CPS applications, research in this area tackles a wide range of issues including visual perception, inference from empirical data, motor learning and control, and the design, implementation, and verification of safe and performant CPS.

Es probable, que sea en base a esta conceptualización que Ercilla ${ }^{32}$ considere el término ciber-físico como aquel que más se acercaría a la descripción de lo que ha de ser un robot inteligente, señalando -en oposición al Parlamento Europeo que hace alusión a una "persona electrónica" - que el término "electrónico" devendría en exiguo a la hora de identificar a los entes dotados de personalidad jurídica y sosteniendo además que a la hora de identificar a los entes dotados de personalidad jurídica la denominación más correcta habría de ser de persona electrofísica ya que este término respondería a la configuración misma de lo que en un futuro serían los robots, a saber, dispositivos físicos con capacidades de computación, almacenamiento y comunicación para controlar e interactuar con un proceso físico, controlados o monitoreados por algoritmos computacionales e integrados en red.

Sin embargo; en base a todas las consideraciones que se recogen y analizan a lo largo del presente trabajo somos de la opinión que un término acertado en este contexto sería "persona artificial antropomórfica".

Si bien debemos aceptar que pocas tecnologías complejas tienen una definición única, estable e incontestada y los robots no son la excepción, refiere Calo ${ }^{33}$ que existe cierto consenso sobre la idea de que los robots son objetos mecánicos que absorben el mundo, procesan lo que sienten y a su vez actúan sobre el mundo resaltando que la utilidad aquí del llamado paradigma sentido-pensar-actuar radica en distinguir a los robots de otras tecnologías ya que la idea de un robot o sistema robótico es que la tecnología combina los tres.

\footnotetext{
${ }^{29}$ El propio Parlamento Europeo señaló al respecto que (...) “un robot podría aprender de forma autónoma de sus experiencias concr etas e interactuar con su entorno de un modo imprevisible y propio únicamente a ese robot".

${ }^{30}$ SEARLE (2000), p. 9.

${ }^{31}$ MAX PLANCK INSTITUTE (2019).

${ }^{32}$ ERCILLA (2018), p. 26.

${ }^{33}$ CALO (2015), p. 529.
} 
Señara Balkin ${ }^{34}$ que Calo lo pone bien cuando habla de "una nueva categoría de sujeto legal a medio camino entre la persona y el objeto". La ubicación es "a medio camino" porque la asignación de estado puede ser incompleta, contextual, inestable y, sobre todo, oportunista. Las personas pueden tratar al robot como una persona (o animal) para algunos propósitos y como un objeto para otros.

Siendo así, esta nueva personalidad jurídica encontrará asidero en la medida que se reconozca y acepte que no estaremos frente a entes sometidos a la total voluntad humana sino que también serán consecuencia del entorno que los rodea lo cual le permitirá adoptar decisiones en virtud de aprendizajes autodidactas y realidades circundantes; de ahí que las implicancias en todo orden de cosas - que acompañarán a esta nueva realidad en la que estamos cada vez más inmersos- avizora un panorama que fue solo concebido por la ciencia ficción de años atrás.

En consideración a los párrafos precedentes somos de la opinión que el primer paso para poder establecer un verdadero Derecho de los Androides ${ }^{35}$ - postulado que consideramos más preciso frente al genérico Derecho de los Robots y más pertinente acorde a los planteamientos y enfoques abordados en el presente trabajo- es la elaboración de códigos de conducta ética que sean de doble observancia, tanto por los ingenieros en el transcurso de sus investigaciones y el proceso de diseño y fabricación, como en el transcurso de la programación específica que se les dará a los robots.

A esto seguirá el establecimiento de una personalidad jurídica propia y la consecuente denominación de persona por la que se optará; bien sea persona electro-física, electrónica, robótica, artificial, sintética o una combinación de estas y por qué no persona artificial antropomórfica, denominación que postulamos en consideración a la importancia que reviste el factor antropomórfico; importancia que ha sido plenamente demostrada en los diversos estudios realizados por reconocidos investigadores en el tema, algunos de los cuales han sido considerados a lo largo del presente trabajo como son Balkin, Calo o Darling; al tiempo que es un factor de reiterada identificación en la amplitud de investigaciones científicas que han abordado el tema; esto en razón a sus múltiples implicancias y el impacto implícito señalado por Turkle o Weinzenbaum.

Sin embargo; al margen de la denominación escogida; determinar la naturaleza de "agente moral" que ostentará esta persona será también un aspecto fundamental ya que difícilmente se podrá hablar de derechos u obligaciones - de diferente índole - atribuibles o exigibles, sin la previa existencia de una "persona".

\section{Vínculos emocionales con objetos electrónicos}

El Parlamento Europeo en la recomendación Nro. 3 del documento ya citado señala que:

(...) que debe prestarse especial atención al posible desarrollo de un vínculo emocional entre seres humanos y robots -especialmente en el caso de grupos vulnerables, como niños, personas mayores

\footnotetext{
${ }^{34}$ BALKIN (2012), p. 57.

${ }^{35}$ Según la RAE un androide es Autómata de figura humana. Sin embargo; algunos usan la palabra robot para referirse a los androides, aunque generalmente se utiliza esta palabra más para referirse a los que se asemejan a humanos, especialmente los que aparentan incluso tener forma, carne y piel humanas. En tanto robot es un término más amplio que considera Máquina o ingenio electrónico programable que es capaz de manipular objetos y realizar diversas operaciones. Que imita las figuras o movimientos de un ser animado. Persona que actúa de manera mecánica o sin emociones. Programa que explora automáticamente la red para encontrar información. Acorde a las definiciones de la RAE, un robot puede ser muchas cosas en estos días desde Roomba pasando por los drones autónomos y los vehículos que técnicamente se conducen a sí mismos; en otras palabras, la lista es interminable, por lo que acorde a esta disimilitud y extensión, no resulta tan simple definir lo que es un robot.
} 
y personas con discapacidad-, y destaca los problemas que pueden plantear las graves consecuencias físicas y emocionales que este vínculo emocional podría causar a los seres humanos.

En ese orden de ideas compartimos la opinión de Balkin al señalar que siempre debemos tener en cuenta los aspectos sociales de la tecnología y el planteamiento de Calo quien afirma que cuanto mejor comprendamos cómo una tecnología afectará a la sociedad, en mejor posición estamos para integrar la tecnología agraciadamente.

Turkle $^{36}$ fue la primera en publicar los efectos de los computadores en la sociedad de manera extensa y profunda; es decir qué nos están haciendo los computadores. La autora, producto de su investigación describió como los propietarios jóvenes de computadores domésticas, algunos expertos programadores, y algunos investigadores en inteligencia artificial llegaron a entablar alguna clase de relación con sus computadores de una cierta forma "original"; siendo estas las primeras formas de relación que muchos propietarios en la actualidad desarrollan con sus mascotas virtuales.

Refirió además que una mascota virtual es la representación en la computadora de un modelo de comportamiento de mascota que al incorporar un software permite a los propietarios interactuar con ella. Esta computadora bien podría ser una PC o una consola de juegos que muestra imágenes de la mascota virtual en su pantalla; podría también ser un producto basado en un microprocesador, tal como un teléfono móvil o un Tamagotchi con una visualización mucho más pequeña que una pantalla de PC; o podría ser un juguete basado en un microprocesador que luce como un animal o un robot. Sin importar su materialización y apariencia, el principio es el mismo: el cerebro virtual de una mascota virtual es simulado por un software en alguna clase de dispositivo de cómputo. En síntesis, el núcleo de una mascota virtual se constituye por una cierta clase de computadora más algún tipo de software.

Las relaciones entre los humanos y las mascotas virtuales son por lo tanto una forma extendida de la relación humano-computador; extendida por la materialización del microprocesador en un diseño de mascota; ya sea que el diseño sea una criatura en una pantalla como un Tamagotchi; el diseño de una muñeca o el cuerpo de una mascota que por sí mismos generan un cierto grado de atracción emocional.

De otro lado - y sin que esté inmersa la tecnología en este caso- es innegable que algunos propietarios de animales valoran la relación con su mascota mucho más que la relación con otras personas, aún con su propia pareja.

Siendo así y en consideración a los contextos señalados nos cuestionamos sobre el porqué no podría esperarse que algunas personas llegaran a expresar sentimientos similares por sus computadores y en consecuencia y por lógica extensión hacia los robots; más aún cuando existen diversos estudios psicológicos sistemáticos que dando lugar a varias teorías en relación al amor por las mascotas identificaron como elemento esencial en estas relaciones el factor antropomórfico y a pesar de que un gran número de psicólogos posiciona este amor en términos de apego las relaciones entre las personas y el mundo animal han ido evolucionando de formas diversas y acorde a las múltiples necesidades humanas; por lo que es innegable que actualmente a una mascota se le considera no solo un miembro más de la familia; sino que también hasta el propietario que lo desee puede contraer matrimonio con ella ${ }^{37}$.

\footnotetext{
${ }^{36}$ Profesora del MIT de Estudios Sociales y Tecnología y Directora del Instituto de Iniciativas en Tecnología y el Ser.

${ }^{37}$ La web site www.marryyourpet.com ofrece la posibilidad de contraer matrimonio con una mascota de forma rápida y fácil —así como lucrativa para su administrador-. El servicio incluye paquetes que están acorde a la elección de una boda simple o una gran boda.
} 
El antropomorfismo ${ }^{38}$ y la tendencia a establecer determinados vínculos sea cual fuere el nombre que se les dé, como podría ser el apego parecieran ser innatos en el ser humano - sin perjuicio de hacia quién o hacia qué se dirija - por lo que Joseph Weinzenbaum, en los inicios de la inteligencia artificial quedo perplejo por el alcance de antropoformización que algunas personas le concedieron al programa de computador ELIZA ${ }^{39}$.

En este orden de ideas refiere Darling ${ }^{40}$ que las personas somos propensas al antropomorfismo; es decir, proyectamos nuestras propias cualidades inherentes en otras entidades para hacerlas parecer más humanas. Una característica clave de los robots sociales es que están diseñados específicamente para generar estas proyecciones.

Compartimos la opinión de la autora al señalar que ya sea por razones psicológicas de proyección sobre otras entidades, conceptos filosóficos morales o preocupaciones prácticas, la regulación del comportamiento violento hacia los objetos robóticos podría estructurarse de manera similar a las leyes de abuso animal que ya tenemos vigentes. En términos de practicidad, un desafío radica en establecer los factores limitantes.

Para aprobar leyes de protección, el primer paso sería definir que es un "robot social" y esta definición en opinión de Darling comprendería a un objeto incorporado con un grado definido de comportamiento autónomo que está específicamente diseñado para interactuar con los humanos a nivel social y responde al maltrato de manera realista tendiendo en consideración además la adaptación a tipos específicos de programación o interacciones sociales específicas. Esto además, conllevaría necesariamente a determinar qué acciones constituirían el "maltrato", lo que en el ámbito tecnológico no solo demandará diversas consideraciones sino que además necesitará reflejar tanto el sentimiento humano como la capacidad tecnológica en contextos específicos.

Esto nos lleva a reflexionar en torno a que es posible que deseemos exigir protección para los robots sociales a fin de desalentar el comportamiento que sería perjudicial en otros contextos y para proteger de cierta forma nuestros valores sociales actuales ya que nuestras acciones hacia los sistemas robóticos reflejarían nuestra moralidad.

Curiosamente el emérito profesor del Instituto Tecnológico de Massachussets Joseph Weinzenbaum afirmaría después que: "El ser humano todavía puede apagar su ordenador. Sin embargo, cada día tendrá que esforzarse más para conservar este privilegio" ${ }^{41}$.

Al mismo tiempo Eric Horvitz ${ }^{42}$ aborda la pérdida de control de los sistemas de IA en unos cien años debido al surgimiento de superinteligencias que no actúen de acuerdo con los deseos humanos señalando que tales sistemas poderosos amenazarían a la humanidad.

\footnotetext{
${ }^{38}$ Es considerado por algunas personas como una creencia, aun que fuere solo transitoria, que el objeto en cuestión es esencialmente humano.

${ }^{39}$ En 1966, Winzenbaum creó el programa Eliza que, junto con su desarrollo de 1967 denominado Parry del psiquiatra Kennet Colby, constituye una sofisticación que va más allá de los juegos de aventura, para adentrarse en la ficción interactiva. Parry simulaba conversaciones de un esquizofrénico paranoico tan fidedignamente que los siquiatras no podían diferenciarlas de las producidas en sus consultas con pacientes reales. Eliza era capaz de sintetizar el significado de varias palabras. La idea de Weizenbaum era que Eliza conversara (de forma escrita) con su interlocutor de modo tal que diera la sensación de que lo estaba escuchando y de que empatizaba con sus problemas e inquietudes para lo cual se valía de diversos recursos. Podía reconocer palabras clave, para luego hacer preguntas referidas a dicho tema; cuando el interlocutor mencionaba en su discurso una palabra, Eliza reconocía que estaba hablando sobre un tema específico y luego hacía preguntas relacionadas con ese tema. También tenía almacenadas frases hechas sobre una variedad de temáticas que utilizaba al reconocer palabras claves así como frases empáticas y de continuidad las cuales siempre resultaban útiles para dar la sensación de estar mostrando interés y hacer que el sujeto continuara hablando como si estuviese manteniendo una conversación real. Si Eliza no encontraba palabras asociadas en su base de datos, empleaba frases y otras combinaciones de palabras; y al igual que hacen muchos psicoterapeutas, daba vuelta la frase que uno le decía y la repetía, transformándola en una pregunta.

${ }^{40}$ DARLING (2016), p. 214.

${ }^{41}$ BAYÉS (2013), p. 28.

${ }^{42}$ HORVITZ (2014), p. 5.
} 


\section{Redimensionando las relaciones interpersonales}

Para Breazeal Cynthia ${ }^{43}$ un robot sociable es capaz de comunicarse, interactuar, comprender y aún relacionarse con nosotros de una forma personal. Al tiempo que debe ser capaz de entenderse y entendernos en términos sociales. Nosotros, a cambio, debemos poder entenderlo en los mismos términos sociales, relacionarnos y ser empáticos con él.

Señala además la autora que este tipo de robot debe poder adaptarse y aprender a lo largo de su tiempo de vida, incorporando experiencias compartidas con otros individuos dentro de su comprensión sobre sí mismo, sobre otros y las relaciones que ellos comparten. En síntesis, según Breazeal un robot sociable es socialmente inteligente de la misma forma que lo es el ser humano e interactuar con él es como interactuar con cualquier otra persona.

Todas las personas se relacionan entre sí y producto de estas relaciones pueden surgir distintas formas o grados de afecto, cercanía, familiaridad u otro lazo. Si bien hemos considerado de manera muy sucinta la relación, vínculo o apego que surge entre las personas y sus mascotas y hemos mencionado el "vínculo emocional" hacia sus mascotas virtuales, incluyendo robots mascotas; entonces no debería sorprendernos que se formen fuertes vínculos emocionales con personas virtuales ${ }^{44}$ o personas robots. Más aún cuando se ha demostrado que en la actualidad existe preferencia por interactuar con computadores que con otras personas. Esta preferencia de interacción explica porque los computadores están teniendo tanto impacto en actividades sociales como la educación, sistema de consejería, psicoterapias, entre otros.

En este orden de ideas, no carece de sentido considerar la posibilidad que surja un vínculo afectivo entre una persona y un robot, vínculo que algunos podrían llamar "amor". Más aún, David Levy ${ }^{45}$ señala que tres distintas secuencias progresivas son las que conducen a un gran número de seres humanos a desarrollar afecto y posteriormente enamorarse, el autor las denomina "rutas para enamorarse de un robot".

Levy refiere que una de estas rutas implica que ellos lleguen a ser cada vez más y más humanos tanto en apariencia como en personalidad lo que motivará nuestro gusto por ellos y poder llegar a amarlos. En realidad; esta ruta es tan solo una extensión natural del amor humano normal como lo conocemos y de las tres rutas consideradas por el autor es la más fácil de comprender. Y como nosotros con el Tamagotchi o con los Furbies, la tendencia humana para cuidar a otros nos generará sentimientos de amor por los robots.

La otra ruta mencionada por Levy, se produce vía amor por las máquinas y por la tecnología per se, comportamiento conocido como "tecnofilia". Esto significa que las personas que "aman" a los computadores lo hacen de diferentes maneras. Es decir, de un lado están aquellos que salen presurosos a comprar cada nuevo dispositivo tecnológico que aparece en el mercado. $Y$ del otro, aquellos para quienes la tecnología se convierte en cierta forma de emoción o más aún en una estimulación erótica, como sería la pornografía en Internet o en algún otro dispositivo. Existen además tecnófilos que son usualmente programadores, pero también están aquellos que aman presionar botones para producir extraños artilugios y cosas extraordinarias; en este caso lo suyo es un amor de control, ya sea el control por escribir los programas que instruyen a sus computadores a hacer algo o; la forma de control más simple obtenida a través de presionar botones en

\footnotetext{
${ }^{43}$ BREAZEAL (2002), p. 1.

${ }^{44}$ Diversos medios de comunicación anunciaron el año pasado que el japonés Akhiko Kondo tuvo una boda única en Tokio al casarse con el holograma de Hatsune Miku, estrella pop de la realidad virtual de 16 años. El holograma se mueve y habla desde un dispositivo de escritorio. Gatebox - la compañía que produce el dispositivo del holograma en el que flota Miku - entregó al novio un certificado de matrimonio de "dimensión transversal".

${ }^{45}$ LEVY (2007), p. 127
} 
dispositivos que ya han sido previamente programados. Más aún, señala Levy que el acto de programar ha sido por sí mismo comparado al sexo en cuanto la programación es una forma de control que somete al computador o al dispositivo al deseo del programador, forzándolo a comportarse conforme a sus deseos, es decir una suerte de "dominación".

La tercera ruta, surgirá a partir de las emociones similares a aquellas que han hecho que las relaciones por Internet sean tal populares y como lo señaló Deb Levine ${ }^{46}$

(...) para algunas personas la atracción en línea y las relaciones llegarán a ser un sustituto válido para la mayoría de relaciones tradicionales. Para aquellos que están recluidos o ruralmente apartados y para aquellos que están aislados de la sociedad por innumerables distintas razones las relaciones en línea podrían convertirse es su única fuente de compañía (...).

Tal como hemos señalado, es común para las personas desarrollar fuertes sentimientos de afecto -incluyendo amor - por sus mascotas (animales); al tiempo que el mismo fenómeno se produce en torno a sus mascotas virtuales. En este orden de ideas, en opinión de Levy además de las tres rutas mencionadas, existen diversos factores que podrían ser importantes para provocar que los humanos se enamoren de los robots. De las diez razones que considera el autor, mencionaremos puntualmente aquellas que podrían encontrar paralelos en las relaciones entre humanos; paralelismos que Levy considera son lo suficientemente fuertes como para desarrollar sentimientos de amor por los robots.

Tenemos así a la Similitud; aunque la personalidad de los robots podría generar escepticismo; es decir ¿cómo podría tener un robot personalidad? A mediados de los años noventa Nass y Moon ${ }^{47}$ del Departamento de Comunicación de la Universidad de Stanford demostraron que es relativamente simple crear características humanas en computadores - personalidades computarizadas - usando una serie de apuntes recogidos de la extensa literatura sobre el tema de la personalidad humana. En términos psicológicos la personalidad es un conjunto de cualidades distintivas que distingue a los individuos. Nass y su grupo ya habían conducido más de treinta y cinco experimentos para investigar algunas de estas cualidades y para poder determinar en qué forma pueden ser simuladas en los programas de computadora y como comparar tales simulaciones con los correspondientes rasgos en los seres humanos ${ }^{48}$.

Si bien diseñar un robot con una personalidad atrayente es un objetivo obvio - además que nos permitiría escoger entre un amplio rango de personalidades, estaturas, apariencia y otras características físicas - surge una pregunta interesante en torno a la necesidad de programar al robot para presentar alguna clase de fricción en su personalidad de modo tal que nos sintamos satisfechos de nuestra relación con ellos al sentir que esas relaciones son genuinas ya que sería muy aburrido que el robot se comportara siempre de la manera esperada. No se buscaría la perfección porque paradójicamente una relación perfecta requiere algunas imperfecciones para crear las sorpresas ocasionales. Siendo así, cuando un humano ordene la compra de un robot deberá completar un cuestionario que recoja información sobre las características deseadas tales como actitudes, religión, creencias, rasgos de la personalidad, hábitos sociales, entre otros; y mientras el robot conozca a su humano cada vez mejor, las características humanas serán observables por el robot que podrá entonces ajustarlas a sus propias características moldeándolas conforme al diseño

\footnotetext{
${ }^{46}$ LEVINE (2000), p. 572.

${ }^{47}$ NASS Y MOON (2000), p. 91.

${ }^{48}$ Uno de los experimentos está relacionado con el elemento "partnership relationship", demostrando que las parejas actúan como equipo de infinidad de formas.
} 
de su humano. En tanto la capacidad de la auto-modificación también permitirá a los robots desarrollar un interés instantáneo en lo que sea que fueren los propios intereses humanos. En síntesis; el propietario encontrará similitud de personalidad en su robot.

Las Características deseables del otro son consideradas también por Levy ya que la palabra clave "deseable" revelada por las investigaciones sobre este tema radica en la personalidad y en la apariencia. Así como la personalidad de un robot puede ser arreglada para soportar cierto grado de similitud a la de su humano también puede ser ajustada para conformar a cualquier tipo de personalidad que su humano encuentre atrayente. Tanto para un robot, como para un humano tener una personalidad ganadora - no obstante programada - será excitante en muchos aspectos; incluyendo el aspecto sexual.

Como se señaló anteriormente la opción de la personalidad del robot puede ser determinada parcialmente antes de su adquisición y una apariencia deseable es aún más fácil de conseguir en un robot. El formato de compra preguntará sobre las dimensiones y las características físicas básicas tales como el peso, color de ojos y cabello, longitud de las piernas, musculatura, entre otros.

En este sentido, la personalidad y la apariencia no son características difíciles para diseñar en los robots como si lo son la síntesis de emociones y personalidad, temas aún en vías de investigación en diversas universidades de los Estados Unidos y otros países. Crear una entidad física en forma humana que sea agradable a la vista es relativamente sencillo y la robot Repliee Q1 diseñada por Hiroshi Ishiguro ${ }^{49}$ es probablemente el primer ejemplo de ello.

La Conexión recíproca es otro de los factores a mencionar. Si consideramos que la reciprocidad en el amor es un elemento importante para que este surja - es decir, es más probable que alguien se enamore de una persona si ya sabe que esta lo ama- el robot que simula demostraciones por su humano lo incentivará a desarrollar sentimientos de amor hacia él. La conexión recíproca, es considerada por Levy como otro atributo que será fácil de replicar en los robots. El robot mostrará entusiasmo por estar en presencia de su propietario; así como por la apariencia y personalidad de este. $Y$ ante la pregunta: ¿realmente le gusto a mi robot? La respuesta probablemente ya la dio Turing en su momento al sustentar su postura sobre la inteligencia de las máquinas; por su parte Levy señala además que la idea de poder gustarle a un robot al inicio podría parecer un tanto espeluznante, pero si el comportamiento de un robot es completamente consistente con la conexión hacia la persona, entonces ¿Por qué deberíamos dudar?

En cuanto a las Influencias sociales afirma Levy que con el tiempo estas sucumben ante un gran cambio. Así, lo que fue considerado una aberración social en los años cincuenta o antes, podría ahora ser considerado como algo normal. Ciertas actitudes hacia los robots cambiarán también con el tiempo en todas las dimensiones y los contextos. Si bien es cierto en la actualidad, según el entorno cultural al que hagamos referencia - oriental u occidental- pueden estar siendo vistos tan solo como juguetes o artículos que despiertan curiosidad, compartimos la opinión de Levy cuando señala que no pasará mucho para que esta visión cambie; para que aquello que ahora es solo curiosidad disminuya hasta desaparecer y se produzca el tránsito que permita que los robots dejen de ser considerados artículos de juego o similares y pasen a ser primero nuestros compañeros, luego nuestros amigos y después nuestros seres queridos. A mayor aceptación se convertirán en nuestros

\footnotetext{
${ }^{49}$ Director del laboratorio de Robótica inteligente del Departamento de Sistemas de Innovación en la Escuela de Postgrado de Cie ncias de la Ingeniería en la Universidad de Osaka. Ha publicado más de 300 artículos en las principales revistas y conferencias como Robótica de Investigación y IEEE PAMI. Así mismo, ha desarrollado muchos humanoides y androides, llamados Robovie, Repliee, Germinoid, Telenoid y Elfoid. En el 2007, Synectics en la Encuesta de Genios Contemporáneos lo distinguió como uno de los 100 principales genios vivos en el mundo actual.
} 
"partners" a menor prejuicio seremos una sociedad que de estar en contra de la existencia de las relaciones humano-robot; nos convertiremos en activistas del gran cambio fomentando el hecho de que más personas acepten tener robots como "partners", amigos y amantes.

En este sentido Levy contempla también entre sus razones el Complementar necesidades; lo que implica que si un robot aprecia las necesidades de su humano será capaz de adaptar su comportamiento adecuadamente, satisfaciendo "esas necesidades". Esto incluye aquellas relaciones en las cuales las necesidades humanas se relacionan con la intimidad y hasta con el sexo. Por lo que otra de sus razones está vinculada a la Excitación, y señala al respecto que este factor depende para su existencia de la situación en la cual un humano y el potencial objeto de amor inicialmente se descubren juntos y no en el objeto de amor por sí mismo. Refiere que el estímulo de excitación es externo a la pareja por lo que resultaría no haber diferencia entre el efecto estimulante que provoca una persona cualquiera al estar ante otra y el efecto que se provoca en esta última cuando está ante la presencia de un robot.

Finalmente Levy esgrime tres razones más que propiciarían que los humanos se enamoren de los robots; una de ellas se basa en lo que tiene a bien llamar Señales específicas por las que se entiende que después de una sesión de prueba y error al adquirir el robot, se podrán identificar exactamente qué tipo características físicas actuarán como señales para provocar el amor de la persona hacia el robot a primera vista; como serían el tipo de voz, fragancia corporal, entre otros elegidos previamente por el adquiriente. Esto podría asimilarse a las características que cualquier persona busca en otra ya sea porque gusta de ellas o son las que suelen serle más atrayentes.

$\mathrm{Y}$ al igual como sucede en las relaciones humanas se requiere que exista la Disposición para entablar una relación, es decir aquella situación en la que se dé cabida a sentimientos de afecto; situación que en el caso de los robots presenta un elemento de Misterio adicional en el entendido que de por sí ya existe este "halo" en torno a ellos, y este se verá incrementado de manera considerable al ir descubriendo como sus facultades mentales y capacidades emocionales se van expandiendo; más aún cuando el comportamiento y desenvolvimiento del robot -como ya señalamos- puede ser dotado de imperfecciones humanas otorgando así al usuario la sensación de superioridad cuando sea beneficiosa en la relación. Este elemento de misterio como sería la "variedad", será un elemento estimulante en la relación humano-robot.

\section{Más allá de un simple experimento mental: La viabilidad de las relaciones humano-robot}

Los diversos cambios que fueron surgiendo en la medida que las sociedades fueron avanzando, trajeron consigo cuestionamientos de todo orden; éticos, jurídicos, sociales, culturales, económicos, políticos, entre otros. Esto llevó a que diversos sectores empezaran a cuestionarse sobre sus realidades; y la pregunta sobre el cómo y el por qué evolucionaron nuestras relaciones personales más importantes aún no encuentra una respuesta definitiva y mucho menos determinante; es más, las posibilidades se incrementan a la par del surgimiento de nuevos avances, elementos o tendencias.

En este sentido; en un mundo donde el matrimonio entre personas del mismo sexo ya ha sido legalizado, donde pueden casarse en línea con sus mascotas o donde simplemente cada uno es lo que quiere ser, o lo que tiene ganas de ser; es natural preguntarse a quién puede incluir el matrimonio en el futuro.

Más aún, si consideramos por un lado que los humanos ya están formando relaciones profundas y significativas con los robots y muchos humanos están intrigados por la posibilidad del romance humano-robot; que va desde el amor platónico hasta la prostitución; y del otro lado 
encontramos que la libertad para contraer matrimonio en casi todos los países es un derecho personal. Siendo así, señalan Goldfeder y Razin ${ }^{50}$ que Hilary Putnam una vez escribió, sobre el concepto de persona robótica, que la "discriminación" basada en la "suavidad" o "dureza" de las partes del cuerpo de un "organismo" sintético parece tan tonto como trato discriminatorio de los humanos sobre la base del color de la piel.

Al mismo tiempo Levy sostiene que los seres humanos ya están involucrados en relaciones sexuales, de cuidado e incluso afectuosas con los robots - la unilateralidad es discutible-.

Si bien, cuán mutuas o consensuadas son estas relaciones en la ficción versus la realidad es un tema diferente y una zona mucho más gris; lo que sí está claro, es que estas relaciones sean del tipo que fueren eventualmente tendrían implicancias legales significativas, por lo que existe una necesidad cada vez mayor de un marco normativo adecuado que las contemple.

Por el contrario, si se considera la opinión del profesor Gary Marchant ${ }^{51}$ : "El matrimonio entre robots y humanos no se trata de derechos de robot; se trata del derecho de un ser humano a elegir casarse con un robot"; las personas que consientan deben tener la posibilidad de entablar cualquier relación matrimonial que elijan libremente, siempre que el matrimonio sea legalmente válido y nadie resulte perjudicado.

En base a todas las posturas consideradas a lo largo del presente trabajo, no resulta ilógico y menos aún difícil de avizorar un escenario en el que humanos y robots puedan contraer matrimonio; más aún cuando sabemos de la actual existencia de relaciones de naturaleza íntima entre ambos - no obstante los cuestionamientos en torno a ellas y que evidentemente nos llevaría a analizar otras circunstancias en determinados contextos-.

\section{Conclusiones}

Cuando los conceptos fundamentales se vuelven problemáticos como marcadores de límites el derecho necesita replantear sus instituciones tradicionales.

No todas las personas jurídicas tienen los mismos derechos y obligaciones, y algunas entidades solo se consideran "personas" para ciertos temas y no para otros. Siendo así; las empresas tienen derecho a un discurso político libre, pero no el derecho al voto. En este sentido si la ley crea personas jurídicas, entonces las "definiciones legales de personas" son prescriptivas más que descriptivas; en consecuencia, llegar a un concepto satisfactorio de la personalidad es una cuestión de decisión, no de descubrimiento. En otras palabras; el establecimiento de la personalidad jurídica corresponde a una evaluación realizada por una legislatura, instancia u órgano ad-hoc a efecto de determinar el otorgamiento derechos y obligaciones a la "entidad"; independientemente de cómo esta se vea y si podría o no pasar por humana o bajo qué consideraciones. A esto se suma el hecho que la noción de personalidad se ha expandido lenta pero significativamente a través de los años y la categoría de personas reconocidas en los tribunales se fue ampliando para viabilizar una tutela jurídica efectiva.

Por lo tanto; en teoría, una persona artificial antropomórfica -o sea cual fuere el nombre que se le confiera - será "portadora" de derechos y obligaciones al tiempo que tendrá múltiples despliegues en diferentes entornos. En consecuencia; las cualidades esenciales de los robots y la IA así como sus indiscutibles repercusiones en el campo del derecho nos conduce a plantearnos la viabilidad de una rama propia que podría denominarse eventualmente: El Derecho de los Androides; consideramos de imperiosa necesidad la existencia de una disciplina autónoma que aunada a su

${ }^{50}$ GOLDFEDER Y RAZIN (2015), p. 148.

${ }^{51}$ MARCHANT (2015). 
carácter multidisciplinario aborde un tratamiento unitario del tema. Este hecho no debe sorprendernos dado que en su momento la irrupción de Internet en nuestras vidas, su desarrollo y evolución; así como las implicancias jurídicas que trajo consigo, significó que el Derecho tuviera que replantearse infinidad de temas jurídicos siendo resultado de ese proceso el nacimiento de una nueva rama del derecho conocido mayoritariamente en nuestro país como Derecho Informático; rama que recibe otros nombres en diferentes países pero cuyo objeto de estudio es sustancialmente el mismo.

El presente estudio ha intentado analizar separadamente las figuras que están inmersas en la "persona" dentro del marco de evolución doctrinaria y las tendencias actuales con la finalidad de justificar la necesidad de creación de una personalidad ad-hoc para los robots; además de esclarecer el hecho que nos encontramos ante un escenario conformado por robots "sociales" que jamás fue concebido por los ordenamientos jurídicos existentes y que debido precisamente a su propia naturaleza y características no logra encajar plenamente en ningún mecanismo de protección tradicional existente; adicionalmente se ha abordado la viabilidad de la existencia de un "agente moral artificial", planteamientos todos que nos conducen a la necesidad y posibilidad de creación de una nueva rama del Derecho.

Por otro lado, debe tenerse presente que en consideración a la naturaleza humana la cual tiende a crear lazos, brindar protección; Ilenar vacíos; satisfacer necesidades, superar frustraciones y demás; y tomando en cuenta las rutas conducentes al amor aunadas a los paralelismos existentes; ante una relación en la cual las "partes" son felices juntas, o al menos manifiestan felicidad eventualmente diversas relaciones interpersonales de naturaleza diferenciadora hasta las ahora conocidas podrían ser viables. En tanto, por ahora no existe ninguna razón por la cual robots y humanos no puedan llevar sus relaciones interpersonales a otro nivel a lo que se suma la falta de consenso normativo en torno a una posible regulación o a la mejor forma de hacerlo y a partir de ese momento asumir las múltiples consecuencias de la decisión que se tome.

\section{BIBLIOGRAFÍA CITADA}

ASHRAFIAN, HUTAN (2014): "AlonAl: A Humanitarian Law of Artificial Intelligence and Robotics", en: Science and Engineering Ethics (Vol. 21, N 1), pp. 29-40.

BALKIN, JACK (2012): “The Path of Robotics Law”, en: California Law Review (Vol. 6), pp. 45-60.

BARRIO, MOISÉS (2018): "Hacia una Personalidad Electrónica para los Robots", en: Revista de Derecho Privado (N²), pp. 89-108.

BAYÉS, RAMÓN (2013): “¿Libertad o Dependencia?”, en: La vanguardia (13 de enero), p. 28.

BREAZEAL, CYNTHIA (2002): Designing Sociable Robots (Massachusetts, MIT Press).

BUSINESS DICTIONARY "Valence". Disponible http://www.businessdictionary.com/definition/valence.html [visitado el 15 de agosto de 2019].

CALO, RYAN (2015): "Robotics and the Lessons of Cyberlaw”, en: California Law Review (Vol. 103, N³), pp. 513-563. 
CAMBRIDGE (2019): "Cambridge Dictionary". Disponible en: https://dictionary.cambridge.org/es/ [visitado el 18 de agosto de 2019].

CAMBRIDGE DICTIONARY (2019a): "Embodiment". Disponible en: https://dictionary.cambridge.org/es/diccionario/ingles/embodiment [visitado el 18 de agosto de 2019].

(2019b): "Emergence". Disponible en: https://dictionary.cambridge.org/es/diccionario/ingles/emergence [visitado el 18 de agosto de 2019].

(2019c): “Unpredictable" Disponible en: https://dictionary.cambridge.org/es/diccionario/ingles-espanol/unpredictable [visitado el 18 de agosto de 2019].

COLLINS DICTIONARY (2019a): "Corporeidad". Disponible en: https://www.collinsdictionary.com/es/diccionario/espanol-ingles/corporeidad [visitado el 19 de agosto de 2019].

(2019b): "Impredecibilidad". Disponible en: https://www.collinsdictionary.com/es/diccionario/espanol-ingles/impredecibilidad [visitado el 20 de agosto de 2019].

DARIO, PAOLO; VERSCHURE, PAUL; PRESCOTT, TONY; CHENG, GORDON; SANDINI, GIULIO; CINGOLANI, ROBERTO; DILLMANN, RÜDIGER; FLOREANO, DARIO; LEROUX, CHRISTOPHE; MACNEIL, SHEILA; ROELFSEMA, PIETER; VERYKIOS, XENOPHON; BICCHI, ANTONIO; MELHUISH, CHRIS Y ALBU, ALIN (2011): "Robot Companions for Citizens", en: Procedia Computer Science (Vol. 7), pp. 47-51.

DARLING, KATE (2016): "Extending legal protection to social robots: The effects of anthropomorphism, empathy, and violent behavior towards robotic objects", en: Calo, Ryan; Froomkin, Michael y Kerr, Ian (Eds.), Robot Law (Massachusetts, Edward Elgar Publishing), pp. 213-231.

ENGINEERING AND PHYSICAL SCIENCES RESEARCH COUNCIL (2010): "Principle of Robotics: Regulating robots in the real world". Disponible en: https://epsrc.ukri.org/research/ourportfolio/themes/engineering/activities/principlesofrobotics/ [visitado el 17 de agosto de 2019].

ERCILLA GaRCíA, JAVIER (2018): Normas de Derecho Civil y Robótica. Robots Inteligentes, Personalidad Jurídica, Responsabilidad Civil y Regulación (Pamplona, Thomson Reuters Arazandi).

GOLDFEDER, MARK Y RAZIN, YOSEF (2015): "Robotic Marriage and the Law", en: LSD Journal (Vol. 10), pp. 137-176.

GROOM, VICTORIA; TAKAYAMA, LEILA; OCHI, PALOMA Y NASS, CLIFFORD (2009): "I Am My Robot: The Impact of Robot-building and Robot Form on Operators", en: HRI'9 Proceedings of the 4th ACM/IEEE international conference on Human robot interaction, pp. 31-36. 
HORVITZ, ERIC (2014): "One Hundred Year Study on Artificial Intelligence: Reflections and Framing". Disponible en: https://ai100.stanford.edu/sites/g/files/sbiybj9861/f/ai100_framing_memo_0.pdf [visitado el 15 de agosto de 2019].

KANT, IMMANUEL (1922): Fundamentación de la Metafísica de las Costumbres (Madrid, Real Sociedad Económica Matritense de Amigos del País).

KAPLAN, JERRY (2017): Abstenerse humanos, guía para la riqueza y el trabajo en la era de la Inteligencia Artificial (Zaragoza, TEELL).

LEVINE, DEB (2000): "Virtual Attraction: What rocks your boat", en: CyberPsychology and Behavior (Vol. 3, $\left.N^{\circ} 4\right)$, pp. 565-573.

LEVY, DAVID (2007): Love and Sex with Robots: The Evolution of Human-Robot Relationships (Nueva York, HarperCollins Publishers).

LINGUEE (2019): “Corporeidad". Disponible en: https://www.linguee.es/espanolingles/search?source=auto\&query=corporeidad [visitado el 3 de agosto de 2019].

MARCHANT, GARY (2015): "Al Thee Wed: Human should be able to marry robots". Disponible en: https://slate.com/technology/2015/08/humans-should-be-able-to-marry-robots.html [visitado el 03 de enero de 2019].

MAX PLANCK INSTITUTE (2019): "Robotics and Cyber-Physical Systems". Disponible en: https://www.cis.mpg.de/robotics/ [visitado el 19 de agosto de 2019].

MOON, YOUNGME Y NASS, CLIFFORD (2000): "Machines and Mindlessness: Social Responses to Computers", en: Journal of Social Issues (Vol. 56, N 1), pp. 81-103.

PAGALLO, UGO (2013): The laws of robots: Crimes, Contracts, and Torts (Países Bajos, Springer).

PARLAMENTO EUROPEO (2017): "Resolución del Parlamento Europeo con recomendaciones destinadas a la Comisión sobre normas de Derecho civil sobre robótica (2015/2103(INL))", en: Diario Oficial de la Unión Europea (16 de febrero de 2017).

REAL ACADEMIA ESPAÑOLA (2019A): "Voluntad". Disponible en: https://dle.rae.es/voluntad [visitado el 10 de agosto de 2019].

(2019B): "Androide". Disponible en: https://dle.rae.es/?id=2abaMxi [visitado el 20 de agosto de 2019].

ROBERTSON, JENNIFER (2014): "Human rights vs. robot rights: Forecasts from Japan", en: Critical Asian Studies (Vol. 46, N4), pp. 571-598.

SEARLE, JOHN (1997): The Mystery of Consciousness (Nueva York, The New York Review of Books). 
SINGER, PETER (2011): Practical Ethics (Cambridge, Cambridge University Press).

TURKLE, SHERRY (2005): The Second Self: Computers and the Human Spirit (Massachusetts, MIT Press).

YUDKOWSKY, ELIEZER (2001): "Creating friendly Al 1.0. The analysis and design of Benevolent goal architecture". Disponible en: https://intelligence.org/files/CFAl.pdf [visitado el 15 de agosto de 2019]. 